\title{
Silencing the expression of MTDH increases the radiation sensitivity of SKOV3 ovarian cancer cells and reduces their proliferation and metastasis
}

\author{
JUN CHEN $^{1}$, YAN JIA ${ }^{1}$, ZAN-HUI JIA $^{1}$, YU ZHU ${ }^{2}$ and YUE-MEI JIN ${ }^{1}$ \\ ${ }^{1}$ Department of Obstetrics and Gynecology, The Second Hospital of Jilin University, Changchun, Jilin 130041; \\ ${ }^{2}$ Department of Ophthalmology, FAW General Hospital, The Fourth Hospital of Jilin University, \\ Changchun, Jilin 130011, P.R. China
}

Received December 12, 2017; Accepted June 12, 2018

DOI: 10.3892/ijo.2018.4541

\begin{abstract}
Ovarian cancer has a high mortality rate among women worldwide. Radiotherapy is considered an effective method of ovarian cancer treatment, however, radioresistance presents a challenge. It is necessary to develop techniques that can increase radiosensitivity in ovarian cancer, and gene therapy is a promising option. The aim of the present study was to investigate the effects of metadherin (MTDH) silencing on the radiosensitivity of ovarian cancer. Ovarian cancer tissues ( $\mathrm{n}=273)$ and normal ovarian tissues $(\mathrm{n}=277)$ were used, as were SKOV3 ovarian cancer cells and the immortalized human ovarian epidermal HOSEpiC cell line. MTT, Transwell and wound-healing assays were performed to assess the proliferation, invasion and migration abilities of the SKOV3 cells. Colony-forming assays and flow cytometry were applied to detect the radiosensitivity and apoptosis of the SKOV3 cells. Nude mouse xenograft models were established to evaluate the effect of MTDH gene silencing on tumor growth and the efficacy of radiotherapy. Ovarian cancer, in tissues and cells, was demonstrated to have a high level of MTDH. Additionally, MTDH silencing was found to significantly inhibit proliferation, migration and invasion, and induce apoptosis in SKOV3 cells, and it was suggested that MTDH depletion significantly increased the sensitivity of the SKOV3 cells to X-ray radiation. MTDH silencing enhanced radiosensitivity and delayed tumor growth in the nude mouse xenograft model. Collectively, the results obtained in the present study suggest the potential role of MTDH silencing as a technique for ameliorating radioresistance in ovarian cancer. The present study provides a
\end{abstract}

Correspondence to: Dr Yue-Mei Jin, Department of Obstetrics and Gynecology, The Second Hospital of Jilin University, 218 Pingzhi Street, Nanguan, Changchun, Jilin 130041, P.R. China

E-mail: jinyuemei_jym@yeah.net

Key words: metadherin, gene silencing, ovarian cancer, radiosensitivity, proliferation, apoptosis promising experimental basis for the improvement of ovarian cancer radiotherapy treatment.

\section{Introduction}

Ovarian cancer has a high mortality rate compared with the majority of types of cancer associated with the female reproductive system (1). The incidence and mortality rates of ovarian cancer have been increasing gradually in China, with at least 230,000 new cases and a mortality rate of $>150,000$ on an annual basis worldwide (2). Of all ovarian cancer cases, $\sim 70 \%$ are detected at an advanced stage, with $85 \%$ of these patients succumbing to the disease $(3,4)$. The risk factors for ovarian cancer include nulliparity and refractory infertility, however, these risks can be mitigated with the use of oral contraceptives for 5 years (5). Surgical intervention in combination with chemotherapy is a common treatment method for ovarian cancer, as ovarian cancer cells are reported to be initially sensitive to chemotherapeutic drugs (6). Although there are a number of treatment modalities available for ovarian cancer, radioresistance is often observed $(7,8)$. Gene therapy has emerged as a novel treatment to overcome radioresistance in ovarian cancer $(9,10)$.

Metadherin (MTDH), also known as astrocyte elevated gene-1 and lysine-rich CEACAM1 co-isolated, is as an important molecule that regulates multiple biological behaviors in carcinogenesis $(11,12)$. It has been suggested that MTDH is associated with chemoresistance and metastasis leading to poor prognoses for patients with breast cancer (13). Initially considered to be a human immunodeficiency virus-induced gene in astrocytes, MTDH is a membrane protein that regulates the homing of tumor cells to the lung endothelium, and is a lysine-rich protein associated with tight junctions in prostate epithelial cells (14). It has been demonstrated that MTDH is upregulated in various types of cancer, including hepatocellular, breast and lung cancer $(11,15,16)$. Additionally, a previous study reported that silencing MTDH markedly improved the radiosensitivity of ovarian cancer and hindered the repair of radiation-induced DNA double strand breaks (17). RNA interference-mediated silencing has been verified to inhibit the expression of therapeutic genes (18). A previous study 
revealed that silencing HOTAIR or mitogen-activated protein kinase 1 inhibited the proliferation, migration and invasion of SKOV3 ovarian cancer cells (19). Therefore, the aim of the present study was to investigate the effects of MTDH silencing on the radiosensitivity, proliferation and metastasis of SKOV3 ovarian cancer cells.

\section{Materials and methods}

Study subjects. Paraffin-embedded ovarian cancer tissues $(n=273)$ were obtained from patients with primary ovarian cancer who were diagnosed at the Second Hospital of Jilin University (Changchun, China) between January, 2012 and March, 2016. The patients (22-59 years of age with a mean age of $39.9 \pm 6.44$ years) had no other medical history or previous history of radio- or chemotherapy. Tumors were histologically confirmed to be serous cystadenoma (124 cases), mucinous cystadenoma (82 cases), endometriod carcinoma (38 cases) and epithelial carcinoma (29 cases; five with mixed epithelial carcinoma, five with clear cell carcinoma and 19 with poorly differentiated carcinoma). All cases were grouped into stage I (35 cases), stage II (78 cases) and stage III (160 cases) according to FIGO2000 (20). Pathological grading was as follows: 48 cases were grade I, 61 cases were grade II and 164 cases were grade III. The histological grading was as follows: 171 cases were poorly differentiated and 102 cases were medium to well differentiated. Lymph node metastasis was present as follows: 172 cases with lymph node metastasis and 101 cases without. For comparison, fresh normal ovarian tissues $(n=277)$ from patients aged 20-56 years old (mean, $36.3 \pm 6.1$ years), who had undergone prophylactic oophorectomy for endometrial carcinoma at the Second Hospital of Jilin University between January 2012 and March 2016, were collected. There was no significant difference in baseline characteristics between the two groups of samples $(\mathrm{P}>0.05)$. The present study was approved by the Ethics Committee of the Second Hospital of Jilin University (no. 201112006) and informed consent was obtained from patients or their guardians in accordance with the Declaration of Helsinki.

Cell culture. The SKOV3 ovarian cancer cells were purchased from the Cell Bank of the Chinese Academy of Sciences (Shanghai, China). The human immortalized ovarian epithelial HOSEpiC cell line (no. BNCC340096; Beina Biotechnology, Co., Ltd., Shanghai, China) was routinely cultured in RPMI-1640 medium (Gibco; Thermo Fisher Scientific, Inc., Waltham, MA, USA) containing $10 \%$ fetal bovine serum in a sterile humidified incubator with $5 \% \mathrm{CO}_{2}$ at $37^{\circ} \mathrm{C}$.

Cell transfection and grouping. MTDH-short hairpin (sh)RNA (interfering plasmid) and control-shRNA (negative plasmid) were synthesized by Shanghai Bioengineering Technology Service Co., Ltd. (Shanghai, China). At $24 \mathrm{~h}$ prior to transfection, the SKOV3 ovarian cancer cells were inoculated into 24-well plates $\left(1.0 \times 10^{5}\right.$ cells/well). The cells were divided into blank, control-shRNA and MTDH-shRNA groups, with three wells in each group, and cultured until cell adherence and confluence (80-90\%) were reached. The transfection reagent kits and transfection reagents used in the experiment were provided by Beijing Kangwei Century Biotechnology Co., Ltd.
(Beijing, China). Only Lipofectamine 2000 was added to the blank group. The appropriate plasmids and transfection reagent were added to the control-shRNA group and MTDH-shRNA group $(0.1 \mu \mathrm{g} ; 0.3 \mu \mathrm{l})$ and suspensions were incubated in RPMI-1640 with $10 \%$ serum for $10 \mathrm{~min}$. Transfection was performed in accordance with the manufacturer's protocol for the transfection reagent.

Reverse transcription-quantitative polymerase chain reaction $(R T-q P C R)$ analysis. The ovarian cancer tissues $(1 \mathrm{~g})$ and total RNA from the SKOV3 cells were treated using TRIzol (Thermo Fisher Scientific, Inc.) according to the manufacturer's protocol. A Transgen RT-PCR kit (Beijing Quanshijin Biotechnology Co., Ltd., Beijing, China) was used to reverse transcribe RNA to cDNA. cDNA was amplified using SYBR-Green (Toyobo, Osaka, Japan) with the specific primers. PCR was initiated with pre-denaturation at $94^{\circ} \mathrm{C}$ for $5 \mathrm{~min}$, followed by 30 cycles of denaturation at $94^{\circ} \mathrm{C}$ for $30 \mathrm{sec}$, annealing $\left(\mathrm{MTDH}, 58^{\circ} \mathrm{C}\right.$; $\beta$-actin, $54^{\circ} \mathrm{C}$ ) for $30 \mathrm{sec}$ and extension at $72^{\circ} \mathrm{C}$ with terminal extension at $72^{\circ} \mathrm{C}$ for $5 \mathrm{~min}$. The primer sequences were as follows: MTDH, forward, 5'-AGCAAAGCAGCCACCA GAG-3' and reverse, 5'-AGGAAATGATGCGGTTGTA-3'; $\beta$-actin, forward, 5'-ACCGAGCGCGGCTACAGC-3' and reverse, 5'-CTCATTGCCAATGGTGAT-3'.

Immunohistochemicalstaining. An SPkit(ZymedLaboratories, San Francisco, CA, USA) was used. Surgical specimens with routine immobilization were paraffin-embedded, cut into 4-m thick sections, dewaxed, heated with a citric acid buffer (pH 6.0; Beijing Nobleryder Technology Co., Ltd., Beijing, China) in a microwave for antigen retrieval (20 min) and stored at room temperature. Endogenous peroxidase was blocked by incubation with $3 \% \mathrm{H}_{2} \mathrm{O}_{2}$ for $10 \mathrm{~min}$. Subsequently, $10 \%$ goat serum (Shanghai Haoran Biotechnology Co., Ltd., Shanghai, China) was used to block samples for $10 \mathrm{~min}$, and primary antibody against MTDH (1:200, PB0309; Wuhan Boster Biological Engineering Co., Ltd., Wuhan, China) was added and incubated overnight at $4^{\circ} \mathrm{C}$. The secondary mouse biotinylated antibody (1:500, BZKT0379; Shanghai Yijisy Co., Ltd., Shanghai, China) was then added and incubated at $37^{\circ} \mathrm{C}$ for $10 \mathrm{~min}$. Horseradish peroxidase (HRP)-labeled streptavidin (Guangzhou Geruilin Biotechnology Co., Ltd., Guangzhou, China) was added at $37^{\circ} \mathrm{C}$ for $10 \mathrm{~min}$ and the tissues were stained with diaminobenzidine. The percentage of positive cells and the staining intensity were scored. A total of five high-power fields were randomly selected in each section (magnification, $\mathrm{x} 400$ ) captured by image analysis software (NikonH600L microscope and image analysis system; Nikon, Tokyo, Japan). The percentage of positive cells was scored as follows: $<5 \%, 0 ; 6-25 \%, 1 ; 26-50 \%, 2 ; 51-75 \%$, 3 ; and $\geq 76 \%, 4$. Staining intensity was scored as follows: 0 (no staining), 1 (yellow), 2 (brown-yellow) and 3 (yellow-brown). The positive cell staining and staining intensity scores were combined and 0-1 was considered as negative (-), 2 as weak positive (+), 3-4 as positive $(++)$ and $\geq 5$ as strong positive $(+++),+,++$ and +++ were considered as positive.

Western blot analysis. PMSF cell lysates were added to the SKOV3 cells. The cells were lysed and centrifuged by $200 \mathrm{~W}$ ultrasonic waves to extract the total protein. Total protein 
concentration was determined using a bicinchoninic acid assay. The proteins (100 g/l) were separated by $12 \%$ SDS-PAGE and transferred onto polyvinylidene fluoride membranes. The membranes were blocked and primary antibodies, including rabbit anti-human MTDH (1:1,000, ab45338) and internal reference $\beta$-actin (1:200, ab8227) (both from Abcam, Shanghai, China), were added and incubated overnight at $4^{\circ} \mathrm{C}$. The membranes were then incubated with HRP-labeled IgG secondary antibody $\left(1: 500\right.$, ab6721; Abcam) at $3^{\circ} \mathrm{C}$ for $2 \mathrm{~h}$, followed by three washes with Tris-buffered saline with Tween-20 (10 min each). All antibodies used were purchased from Santa Cruz Biotechnology, Inc., (Dallas, TX, USA). The samples were developed using a chemiluminescence system. The gray value was assessed using a grayscale scanner and the relative protein expression was calculated using ImageJ software.

MTT assay. A cell suspension was prepared from SKOV3 cells from the three groups in the logarithmic growth phase, and the density was adjusted to $3 \times 10^{4}$ cells $/ \mathrm{ml}$. Subsequently, $100 \mu \mathrm{l}$ cell suspension was seeded into 96-well plates with five wells for each group. At 24, 48 and $72 \mathrm{~h}$ post-transfection, MTT solution $(20 \mu \mathrm{l}$ per well) was added. Following $4 \mathrm{~h}$ of incubation in the dark, the supernatant was aspirated. Dimethylsulfoxide $(150 \mu \mathrm{l})$ was added to each well. The absorbance values were measured at $570 \mathrm{~nm}$ using a microplate reader. The optical density (OD) values were used to calculate the in vitro proliferation rate of each group.

Transwell assay. Matrigel was homogenized and added to the upper chambers of a Transwell chamber (Corning Incorporated, Corning, NY, USA). Following $6 \mathrm{~h}$ of transfection, the SKOV3 cells were digested and centrifuged $(300 \mathrm{x} \mathrm{g}$, $3 \mathrm{~min}, 37^{\circ} \mathrm{C}$ ) for further use. The cell suspensions from the blank, control-shRNA, and MTDH-shRNA groups were seeded into Transwell chambers in 24-well plates with chemokines and culture medium. The chambers were incubated at $37^{\circ} \mathrm{C}$ in an atmosphere containing $5 \% \mathrm{CO}_{2}$ for $24 \mathrm{~h}$. A total of five high-power fields were observed under a microscope (Olympus) to calculate the number of cells penetrating the membrane.

Wound-healing assay. The SKOV3 cells were seeded into 6-well plates with three wells for each group. The SKOV3 cells were grown until $\sim 90 \%$ density was reached, following which the culture medium was aspirated and a pipette tip was used to create a number of parallel scratches in the middle of culture wells. The cells were washed and continually cultured. A total of nine fields with scratches were randomly selected in each group, and images were captured at 0, 24, 48 and $72 \mathrm{~h}$ under a microscope (Olympus). Image-Pro Plus 6.0 (Media Cybernetics, Inc., Rockville, MD, USA) was used to measure the distance between two scratches and calculate the rates of cell migration. Cell migration $=(\mathrm{scratch}$ distance at $0 \mathrm{~h}$ - scratch distance at 24,48 or $72 \mathrm{~h}$ )/scratch distance at $0 \mathrm{~h}$.

Colony-forming assay. According to different doses of irradiation, cells were inoculated into culture dishes $(6 \mathrm{~cm})$. When the cells had adhered, the culture dishes were irradiated with a single dose of $\mathrm{X}$-rays at $0,2,4,6$ and 8 Gy respectively, and the irradiation was performed at room temperature at the dose rate of $2 \mathrm{~Gy} / \mathrm{min}$. Following irradiation, three parallel samples were incubated for 2 weeks and stained using Giemsa. The number of colonies (clusters of $\geq 50$ cells) was counted using a microscope and the experiment was performed in triplicate. Planting efficiency $(\mathrm{PE})=$ number of clones/number of inoculated cells X100 (\%).

Flow cytometry. When the cells had been centrifuged and trypsinized, the final concentration of the single cell suspension was adjusted to $1.25 \times 10^{5}$ cells $/ \mathrm{ml}$. Blank, control-shRNA and MTDH-shRNA cell suspensions were inoculated into 6 -well plates $\left(1.25 \times 10^{5}\right.$ cells/well $)$. The cells were exposed to irradation at $0,2,4,6$ and $8 \mathrm{~Gy}$, which was performed at room temperature at the dose rate of $2 \mathrm{~Gy} / \mathrm{min}$. The samples were washed twice with PBS following irradiation and cell suspensions were collected in the corresponding flow tubes following digestion with $0.25 \%$ trypsin. Binding buffer $(500 \mu \mathrm{l})$ and Annexin V-FITC (5 $\mu \mathrm{l})$ were added to each tube and mixed well, followed by the addition of propidium iodide (5 $\mu \mathrm{l})$ and further mixing. The samples were incubated at room temperature for $15 \mathrm{~min}$ in the dark. The apoptotic rate was measured by flow cytometry for $1 \mathrm{~h}$, and the experiment was performed in triplicate. The above reagents were purchased from BD Biosciences (San Jose, CA, USA).

Nude mouse xenograft. Nude mouse xenograft models were established using female BALB/c-nude mice (4-6 weeks old, weighing 16-20 g; n=30; Beijing Weitong Lihua Co., Beijing, China), which were randomly divided into three groups (10 in each group; 17-18 g) and maintained in a specific pathogen-free (SPF) 'barrier' facility with controlled at room temperature and 55-62\% humidity, and alternating 12-h light and dark cycles, food and water is accessible. The SKOV3 cells in the logarithmic growth phase were extracted from the blank, control-shRNA and MTDH-shRNA groups and cell suspensions were produced $\left(4 \times 10^{7}\right.$ cells $\left./ \mathrm{ml}\right)$. Subsequently, $0.3 \mathrm{ml}$ of cell suspension was inoculated into the subcutaneous tissue above the right scapula of mice in each group, and tumor formation was induced after 10 days. When the tumor size was $100 \mathrm{~mm}^{3}$, all female BALB/c-nude mice were anesthetized with $3 \%$ pentobarbital sodium $(30 \mathrm{mg} / \mathrm{kg}$ intraperitoneally; P3761; Sigma-Aldrich; Merck Millipore, Darmstadt, Germany). All mice were placed in a lead box following anesthesia. The right scapula was fixed outside the lead box and locally irradiated with X-rays every other day; irradiation was performed at room temperature at the dose rate of $2 \mathrm{~Gy} / \mathrm{min}$ each time (five times), with the total dose of 10 Gy (MultiRad225; Faxitron X-ray Corporation, Wheeling, IL, USA). A total of 30 female BALB/c-nude mice were sacrificed following X-ray irradiation for 28 days. Immediately after sacrifice, body weight was detected. No significant difference was found in body weight of all BALB/c-nude mice, as all mice weighed approximately $24 \mathrm{~g}$. The gross morphology and growth of the nude mouse xenografts were examined and recorded carefully. The stripped tumor was washed with $100 \mu 1$ sterile water four times. The experiment was approved by the Animal Ethics Committee of the Second Hospital of Jilin University (no. 201201003), and the experiment strictly followed the National Institutes of Health guide for the care and use of laboratory animals. 

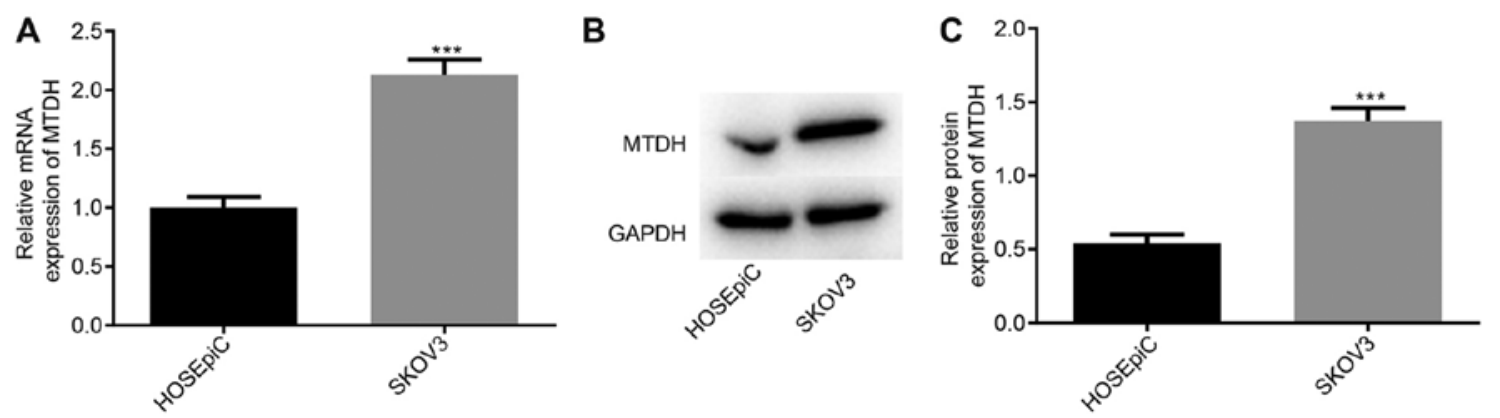

Figure 1. Expression of MTDH is increased in SKOV3 cells. (A) Reverse transcription-quantitative polymerase chain reaction and (B and C) western blot analyses were used to determine the relative expression of MTDH in SKOV3 and HOSEpiC cells. ${ }^{* * *} \mathrm{P}<0.05$, vs. HOSEpiC cells. Data are presented as the mean \pm standard deviation and were analyzed using Student's t-test. The experiment was repeated three times. MTDH, metadherin.

A

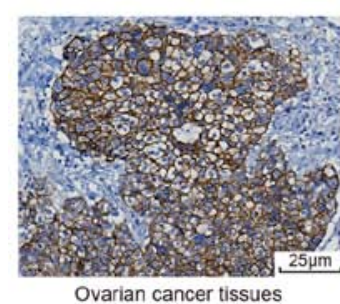

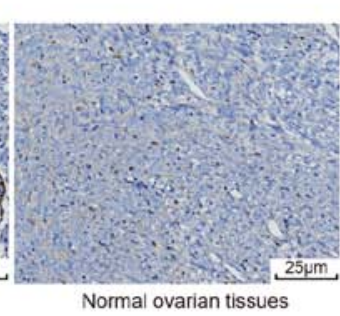

B

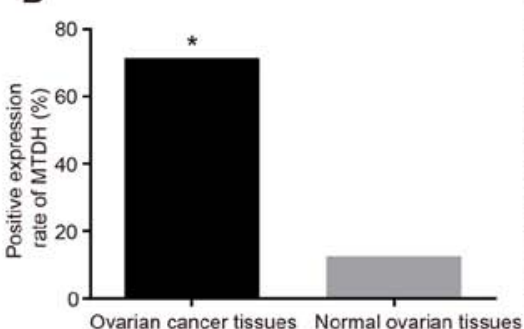

C

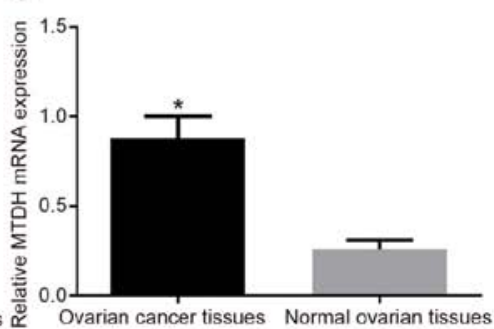

Figure 2. Expression of MTDH is high in ovarian cancer tissues. (A) Immunohistochemistry was used to assess the relative expression of MTDH in ovarian cancer tissues and normal ovarian tissues (SP magnification, x400). (B) Expression of MTDH was elevated in ovarian cancer tissues. (C) Reverse transcriptionquantitative polymerase chain reaction analysis was used to determine the relative expression of MTDH in ovarian cancer tissues and normal ovarian tissues; ${ }^{*} \mathrm{P}<0.05$ vs. normal ovarian tissues. There were 273 cases of ovarian cancer and 277 normal cases. Data are presented as the mean \pm standard deviation and analyzed by Student's t-test. The experiment was repeated three times. MTDH, metadherin.

Statistical analysis. All data were processed using SPSS 20.0 software (IBM SPSS, Armonk, NY, USA) and are presented as the mean \pm standard deviation. Student's t-test was used for comparisons between two groups and one-way analysis of variance was used to compare multiple groups. The association between MTDH expression, ovarian cancer and clinicopathological factors in ovarian cancer was assessed using the $\chi^{2}$ test. For homogenous data, the Least Significant Difference test was performed; for non-homogenous data, the Games-Howell test was used. $\mathrm{P}<0.05$ was considered to indicate a statistically significant difference.

\section{Results}

MTDH is expressed at high levels in SKOV3 cells. To measure the expression of MTDH in SKOV3 cells, RT-qPCR and western blot analyses were performed. The results demonstrated that the expression of MTDH was increased in SKOV3 cells compared with that in HOSEpiC cells $(\mathrm{P}<0.05$; Fig. 1$)$. These results suggested that MTDH is associated with ovarian cancer.

MTDH is expressed at high levels in ovarian cancer tissues. Subsequently, the expression of MTDH in ovarian cancer tissues was examined using immunohistochemistry and RT-qPCR analysis. The cytoplasm or cell membrane was stained brown-yellow in positive cells, indicating MTDH expression. The positive rate of MTDH expression in ovarian cancer tissues was $71.43 \%$, compared with $12.64 \%$ in normal ovarian tissues $(\mathrm{P}<0.05$; Table I). More positive cells were expressed in the ovarian cancer tissues than in the normal ovarian tissues ( $\mathrm{P}<0.05$; Fig. 2A and B). The RT-qPCR results demonstrated that the expression of MTDH was significantly elevated in ovarian cancer tissues compared with normal ovarian tissues $(\mathrm{P}<0.05$; Fig. $2 \mathrm{C})$. The association between the expression of MTDH and clinicopathological features in ovarian cancer tissues is shown in Table II; the results suggested that the expression of MTDH was associated with clinical stage, pathological grade, histological grade and lymph node metastasis. No significant differences were observed between histological subtype and age (Table II).

MTDH silencing significantly reduces the expression of $M T D H$. RT-qPCR and western blot analyses were performed to evaluate the mRNA and protein expression of MTDH in SKOV3 cells following transfection in each group. Compared with the blank and control-shRNA groups, SKOV3 cells in the MTDH-shRNA group had decreased mRNA and protein levels of MTDH $(\mathrm{P}<0.05)$, whereas no significant differences in expression were observed in the blank and control-shRNA groups $(\mathrm{P}>0.05)$. This suggested successful shRNA-mediated inhibition of MTDH in theSKOV3 cells (Fig. 3).

MTDH silencing significantly suppresses ovarian cancer growth. The viability of SKOV3 cells was assessed using an MTT assay (Fig. 4). The respective OD values in the blank, control-shRNA and MTDH-shRNA groups were $0.63 \pm 0.09$, $0.67 \pm 0.08$ and $0.40 \pm 0.04$ at 1 day, $1.33 \pm 0.11,1.47 \pm 0.13$ and 
Table I. Expression of MTDH in ovarian cancer tissues and normal ovarian tissues.

\begin{tabular}{lcrrrrr}
\hline & & \multicolumn{2}{c}{ MTDH expression } & & \\
\cline { 3 - 4 } Group & $\mathrm{n}$ & Positive & Negative & & Positive rate (\%) & P-value \\
\hline Normal ovarian tissues & 277 & 35 & 242 & 12.64 & 0.001 \\
Ovarian cancer tissues & 273 & 195 & 78 & 71.43 & \\
\hline
\end{tabular}

The P-value was evaluated using the $\chi^{2}$ test. MTDH, metadherin.

Table II. Correlation between the expression of MTDH and the clinicopathological features of ovarian cancer.

\begin{tabular}{|c|c|c|c|c|c|}
\hline \multirow[b]{2}{*}{ Clinicopathological factor } & \multirow[b]{2}{*}{$\mathrm{n}$} & \multicolumn{2}{|c|}{ MTDH expression } & \multirow[b]{2}{*}{$\chi^{2}$ value } & \multirow[b]{2}{*}{ P-value } \\
\hline & & Positive & Negative & & \\
\hline \multicolumn{6}{|l|}{ Histological classification } \\
\hline Serous cystadenocarcinoma & 124 & 90 & 34 & 1.982 & 0.576 \\
\hline Mucinous cystadenocarcinoma & 82 & 55 & 27 & & \\
\hline Endometrioid carcinoma & 38 & 30 & 8 & & \\
\hline Epithelial carcinoma & 29 & 20 & 9 & & \\
\hline \multicolumn{6}{|l|}{ FIGO stage } \\
\hline I, II & 113 & 54 & 59 & 52.800 & $<0.001$ \\
\hline III & 160 & 141 & 19 & & \\
\hline \multicolumn{6}{|l|}{ Grade } \\
\hline $\mathrm{I}, \mathrm{II}$ & 109 & 52 & 57 & 50.030 & $<0.001$ \\
\hline III & 164 & 143 & 21 & & \\
\hline \multicolumn{6}{|l|}{ Histology } \\
\hline Poor differentiation & 171 & 146 & 25 & 43.650 & $<0.001$ \\
\hline Middle or high differentiation & 102 & 49 & 53 & & \\
\hline \multicolumn{6}{|l|}{ Age (years) } \\
\hline$\geq 40$ & 145 & 98 & 47 & 2.237 & 0.135 \\
\hline$<40$ & 128 & 97 & 31 & & \\
\hline \multicolumn{6}{|l|}{ Lymph node metastasis } \\
\hline No & 101 & 47 & 54 & 48.680 & $<0.001$ \\
\hline Yes & 172 & 148 & 24 & & \\
\hline
\end{tabular}

The P-value was evaluated using the $\chi^{2}$ test. MTDH, metadherin.

$0.93 \pm 0.06$ at 2 days, and $1.59 \pm 0.12,1.67 \pm 0.13$ and $1.02 \pm 0.11$ at 3 days of tumor growth. The respective OD values in the blank, control-shRNA and MTDH-shRNA groups were $1.81 \pm 0.07$, $1.86 \pm 0.11$ and $1.18 \pm 0.08$ at 5 days, and $1.94 \pm 0.12,1.97 \pm 0.12$ and $1.23 \pm 0.09$ at 7 days of tumor growth. The cell growth in the MTDH-shRNA group was inhibited compared with that in the blank and control-shRNA groups at each time point following transfection $(\mathrm{P}<0.05)$, whereas no significant differences were observed in the blank and control-shRNA groups $(\mathrm{P}>0.05)$. These results suggested that the shRNA-mediated inhibition of MTDH suppressed the proliferation of SKOV3 cells.

MTDH-shRNA suppresses the migration and invasion of ovarian cancer SKOV3 cells. The effects of MTDH-shRNA transfection on ovarian cancer cell migration and invasion were determined using Transwell and wound-healing assays. The Transwell results revealed no significant differences in the number of cells penetrating the membrane between the blank (44.67 \pm 4.51$)$ and control-shRNA (41.67 \pm 5.51$)$ groups $(\mathrm{P}>0.05)$. Compared with the blank and control-shRNA groups, a significantly lower number of cells $(7.00 \pm 2.00)$ penetrated the membrane in the MTDH-shRNA group $(\mathrm{P}<0.05$; Fig. 5A and $\mathrm{B})$. The wound-healing assay revealed that cell migration was significantly reduced at each time point in the MTDH-shRNA group compared with migration in the blank and control-shRNA groups $(\mathrm{P}<0.05$; Fig. 5C and D). These results suggested that shRNAmediated inhibition of MTDH suppressed the migration and invasion of SKOV3 cells. 

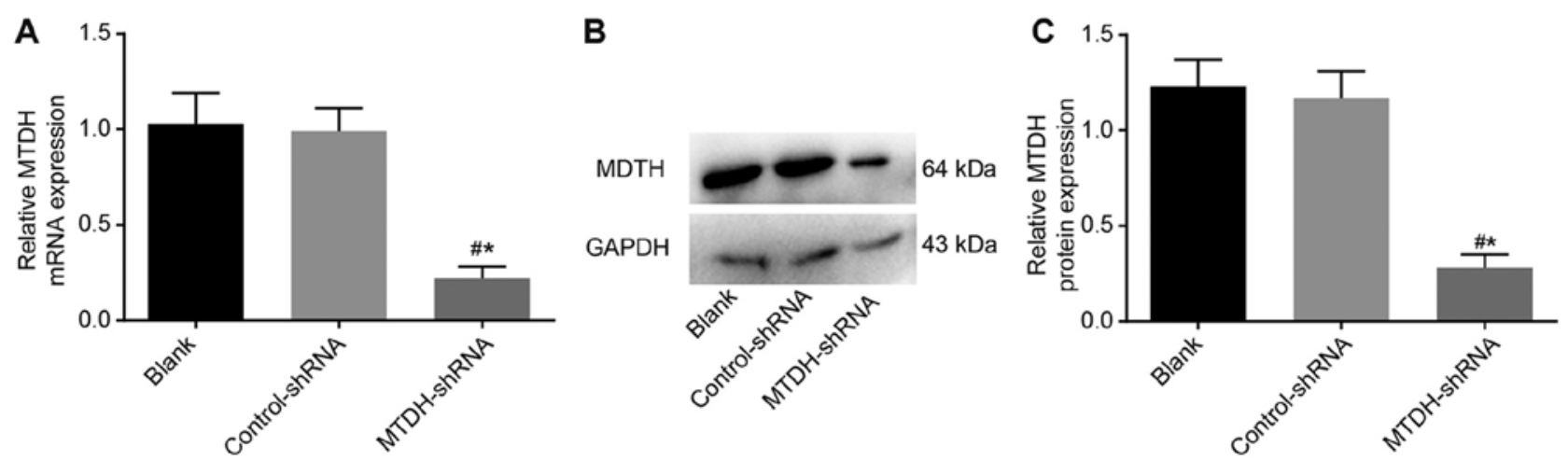

Figure 3. SKOV3 cells have a high expression of MTDH. (A) Reverse transcription-quantitative polymerase chain reaction and (B) western blot analyses were used to determine the relative expression of MTDH in each group, with $(\mathrm{C})$ quantification of western blot results. ${ }^{*} \mathrm{P}<0.05$, vs. blank group; ${ }^{*} \mathrm{P}<0.05$ vs. controlshRNA group. Data are presented as the mean \pm standard deviation and were analyzed by one-way analysis of variance. The experiment was repeated three times. MTDH, metadherin; shRNA, short hairpin RNA.

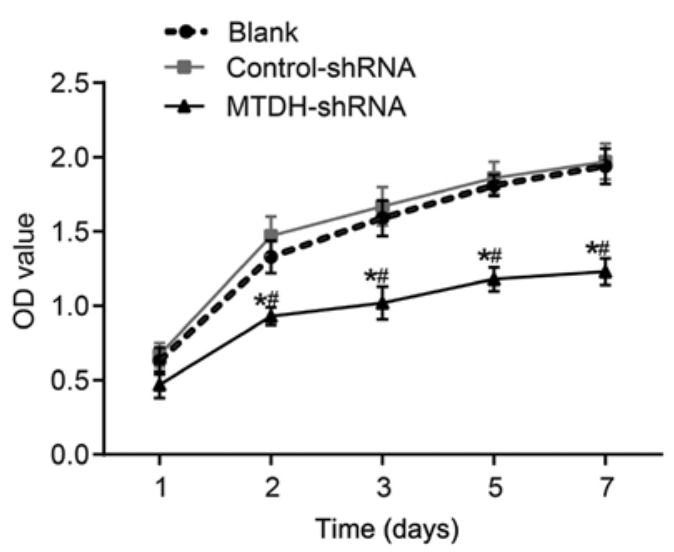

Figure 4. MTDH silencing inhibits the viability of SKOV3 cells. An MTT assay was used to assess viability. ${ }^{*} \mathrm{P}<0.05$ vs. MTDH-shRNA group; ${ }^{\text {"P }}<0.05$ vs. 0 Gy. Data are presented as the mean \pm standard deviation and were analyzed by one-way analysis of variance. The experiment was repeated three times. MTDH, metadherin; shRNA, short hairpin RNA; OD, optical density.

$M T D H$ gene silencing increases the radiosensitivity of SKOV3 cells. In order to analyze the effect of MTDH gene silencing on the radiosensitivity of SKOV3 cells, a colonyformation assay was performed. The colony-formation assay is regarded as a standard measurement to evaluate the effect of external radiotherapy on tumors by measuring the reproductive integrity of tumor cells without proliferation (21). The cell survival fraction was decreased in the blank, controlshRNA transfection and MTDH-shRNA groups depending on the radiation dose $(\mathrm{P}<0.05)$. The surviving fraction was lower in the MTDH-shRNA group compared with that in the blank and control-shRNA groups (all $\mathrm{P}<0.05$ ), whereas no significant differences in the cell survival fraction were observed between the blank and control-shRNA groups ( $P>0.05$; Fig. 6). Taken together, these results indicated that MTDH silencing increased the sensitivity of the SKOV3 cells to $\mathrm{X}$-ray radiation.

MTDH silencing increases the apoptosis of SKOV3 cells following radiotherapy. Flow cytometry was used to assess the apoptotic rate following irradiation (Fig. 7). The variance analysis indicated that apoptosis was increased with increasing doses of radiation in the blank, control-shRNA and MTDHshRNA groups $(\mathrm{P}<0.05)$. The apoptotic rate was significantly increased in the MTDH-shRNA group compared with that in the blank and control-shRNA groups $(\mathrm{P}<0.05)$, whereas no significant difference was observed between the blank and control-shRNA groups $(\mathrm{P}>0.05)$. These results suggested that MTDH silencing enhanced radiotherapy-induced apoptosis of SKOV3 cells.

MTDH silencing inhibits tumor growth and enhances the radiosensitivity of SKOV3 cells. A nude mouse xenograft model was utilized to assess differences in tumor growth following inoculation with SKOV3 cells. The growth of xenograft tumors was observed in nude mice as the efficacy of radiotherapy. Slow tumor growth was observed for 8 days prior to radiotherapy in the blank, control-shRNA and MTDHshRNA groups, and no significant differences in tumor volume were observed $(\mathrm{P}>0.05)$. On day 12 of radiotherapy, the tumor volume was $110.51 \pm 9.73$ and $113.72 \pm 8.96 \mathrm{~mm}^{3}$ in the blank and control-shRNA groups, respectively, with no significant differences observed $(\mathrm{P}>0.05)$. In the MTDH-shRNA group, tumor volume was $90.13 \pm 6.92 \mathrm{~mm}^{3}$, indicating a significant decrease in tumor growth rate compared with that in the blank and control-shRNA groups $(\mathrm{P}<0.05)$. Radiotherapy was continued, and the tumor volume in the MTDH-shRNA group decreased compared with that in the blank and controlshRNA groups $(\mathrm{P}<0.05)$. The mice were sacrificed at the end of radiotherapy and the tumor tissues were weighed. The mean tumor weights in the blank, control-shRNA and MTDHshRNA groups were $1.27 \pm 0.14,1.25 \pm 0.17$ and $0.57 \pm 0.10 \mathrm{~g}$, respectively. The mean tumor weight was decreased in the MTDH-shRNA group compared with that in the blank and control-shRNA groups $(\mathrm{P}<0.05)$, however, no significant differences were observed between the blank and controlshRNA groups ( $\mathrm{P}>0.05$; Fig. 8A-C). These results suggested that MTDH silencing enhanced the radiosensitivity of the nude mouse xenograft models and effectively delayed tumor growth. Furthermore, the protein level of MTDH was reduced in the MTDH-shRNA group compared with that in the blank and control-shRNA groups $(\mathrm{P}<0.05)$, whereas no such difference was observed between the blank and control-shRNA groups ( $\mathrm{P}>0.05$; Fig. $8 \mathrm{D}$ and $\mathrm{E})$. 
A

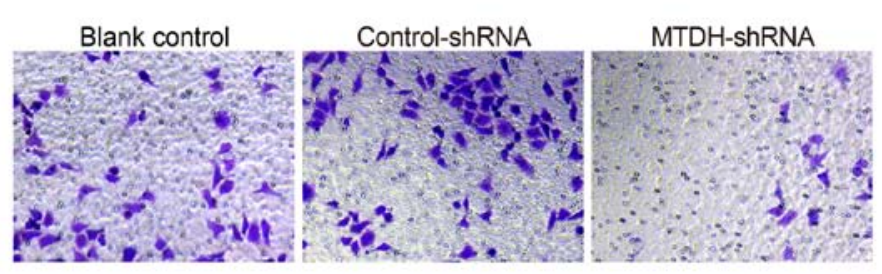

C
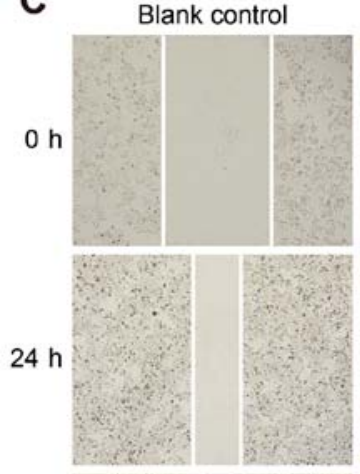

$48 \mathrm{~h}=(\mathrm{x})$

$72 \mathrm{~h} \cdots(x)$
Control-shRNA
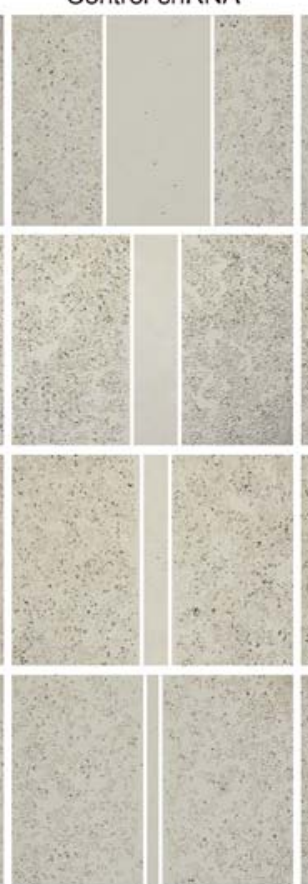

MTDH-ShRNA
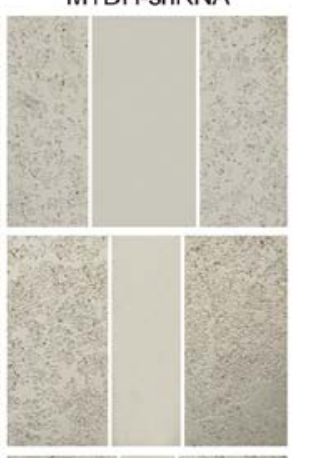

B

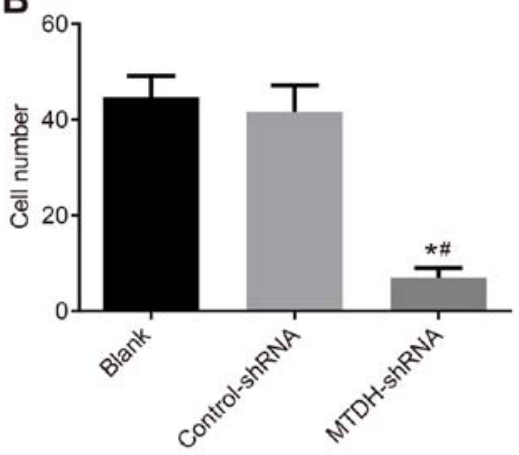

D

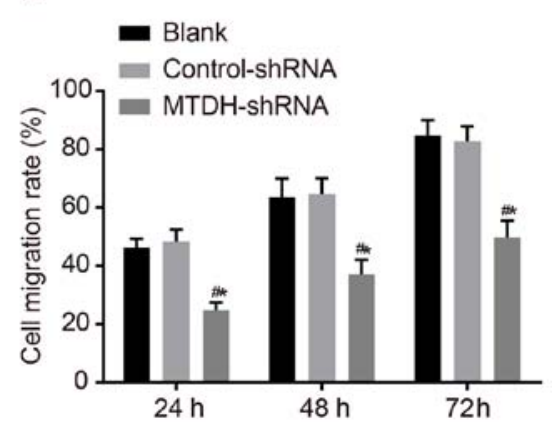

Figure 5. MTDH silencing suppresses the invasion and migration of SKOV3 cells. Transwell and wound-healing assays were performed. (A) Images and (B) quantification of invasion, and the (C and D) results of migration showed that invasion and migration were inhibited by MTDH depletion. ${ }^{*, *} \mathrm{P}<0.05 \mathrm{vs}$. blank and control groups. Data are presented as the mean \pm standard deviation and were analyzed by one-way analysis of variance. The experiment was repeated three times. MTDH, metadherin; shRNA, short hairpin RNA. Original magnification, x200.

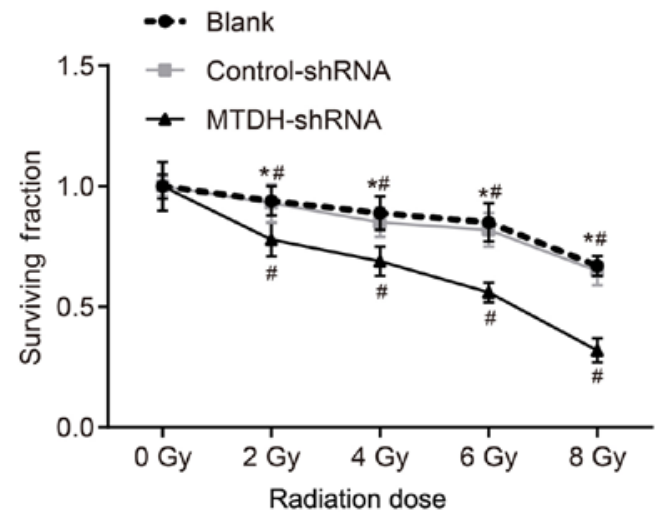

Figure 6. SKOV3 survival fractions decrease with irradiation in a dosedependent manner. Data are presented as the mean \pm standard deviation and were analyzed by one-way analysis of variance. ${ }^{*} \mathrm{P}<0.05$ vs. MTDH-shRNA group; ${ }^{\#} \mathrm{P}<0.05$ vs. 0 Gy. The experiment was repeated three times. MTDH, metadherin; shRNA, short hairpin RNA.

\section{Discussion}

Ovarian cancer is a type of cancer that affects the female reproductive tract and is the fifth-leading cause of cancer-associated mortality among women (22). In the last decade, MTDH has been identified as an important oncogene and is a valuable prognostic marker in patients with various types of cancer. It has been reported that MTDH is localized in the cell membrane, cytoplasm, endoplasmic reticulum, nucleus and nucleolus (23-25). The application of MTDH gene silencing to overcome radioresistance in ovarian cancer is of great interest. The present study investigated the effects of MTDH silencing on the radiosensitivity, proliferation, migration, invasion and apoptosis of SKOV3 ovarian cancer cells. The results indicated that MTDH silencing inhibited cell proliferation, migration and invasion, and promoted cell apoptosis and radiosensitivity in vitro and in vivo.

The expression of MTDH was assessed in ovarian cancer and normal ovarian tissues, and in ovarian cancer and normal cell lines. The results demonstrated that the expression of MTDH was high in ovarian cancer tissues compared with that in normal tissues, and that transfection with MTDH-shRNA reduced the expression of MTDH in the SKOV3 ovarian cancer cells. The association between MTDH and oncology has been investigated in several types of cancer, providing an insight into factors affecting prognoses (26-28). It has been reported that MTDH is expressed in human ovarian cancer 
A
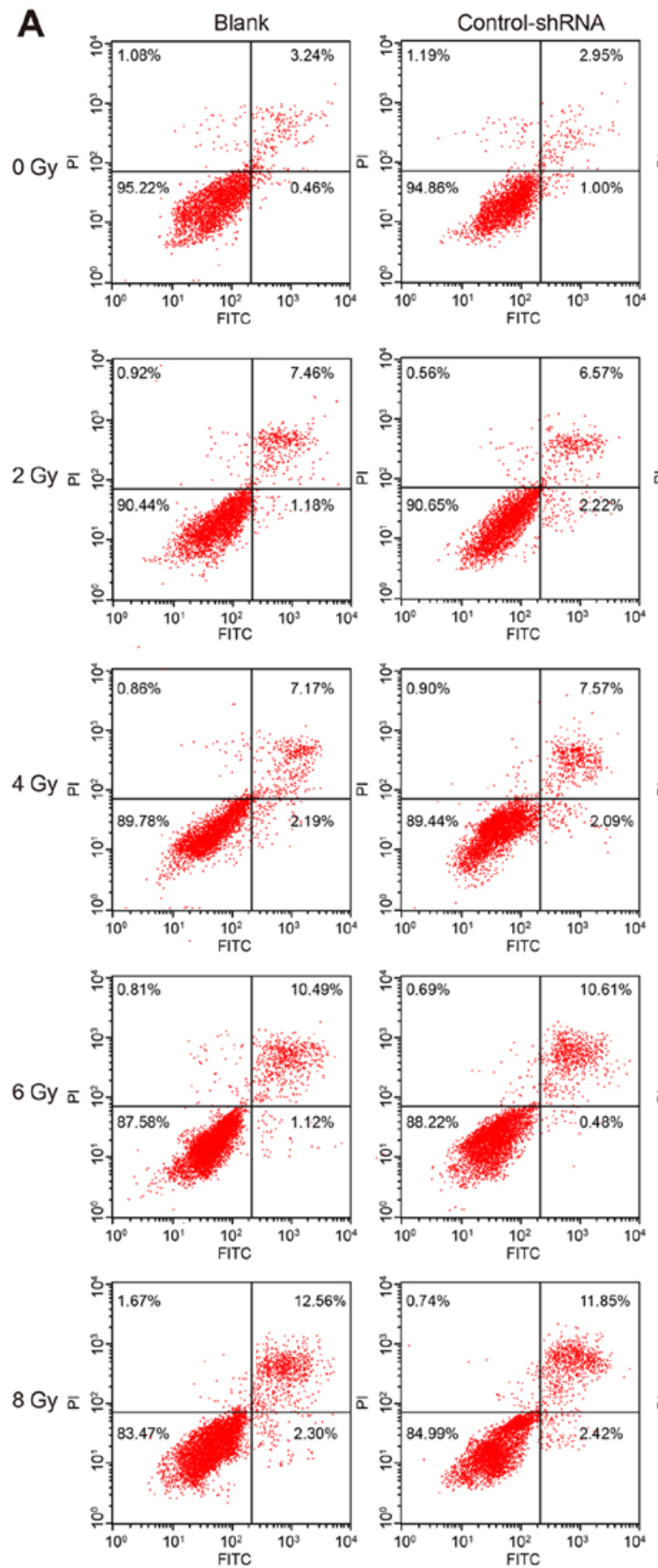
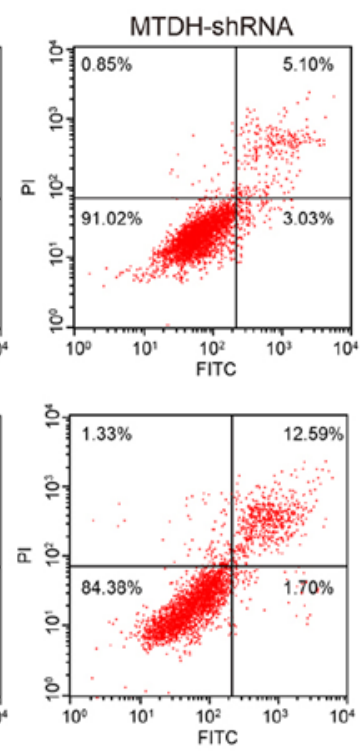

B
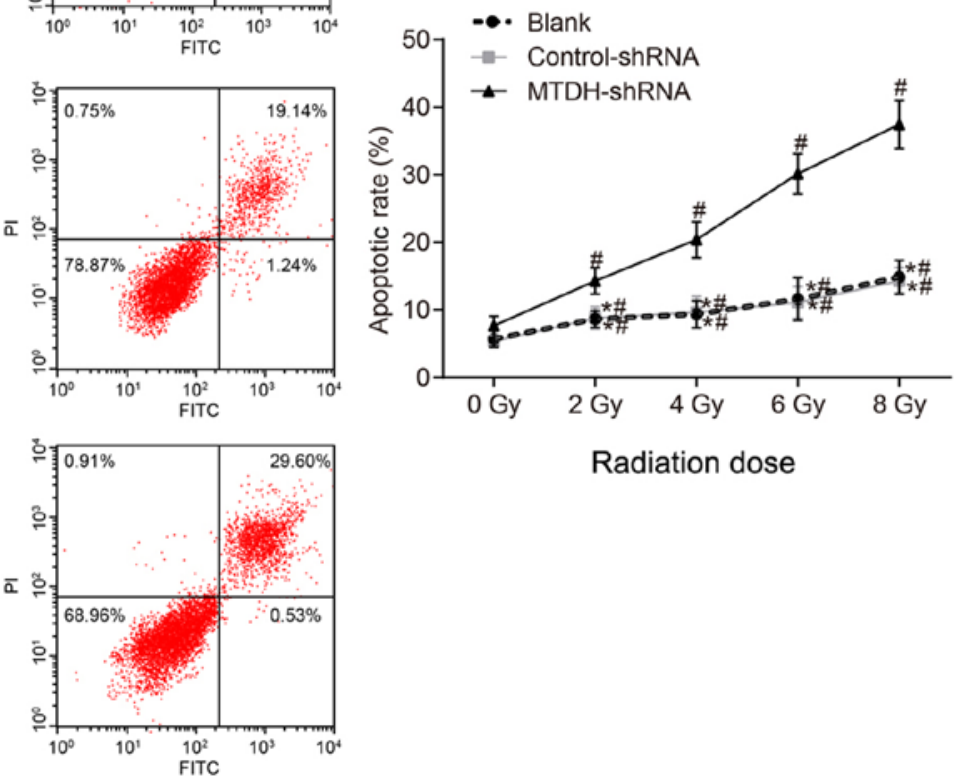

Radiation dose

Figure 7. MTDH gene silencing increases the radiosensitivity of SKOV3 cells. (A) Flow cytometry revealed that the apoptosis was increased with irradiation in a dose-dependent manner. (B) Apoptosis was promoted by MTDH depletion. ${ }^{*} \mathrm{P}<0.05$ vs. the MTDH-shRNA group; ${ }^{\text {P }}<0.05$ vs. 0 Gy. Data are presented as the mean \pm standard deviation and were analyzed by one-way analysis of variance. The experiment was repeated three times. MTDH, metadherin; shRNA, short hairpin RNA; PI, propidium iodide.

tissues and is negatively correlated with the overall survival rate of patients (29). The upregulation of MTDH is frequently observed in various types of cancer, including liver, brain and breast cancer, and contributes to poor prognoses $(28,30)$. Consistent with the results of the present study, Zhou et al (31) reported that there was minimal or no MTDH immunoreactivity in normal tissues. Similarly, the overexpression of MTDH is associated with the prognosis of patients with metastatic ovarian cancer, and MTDH staining was increased in chemoresistant patients compared with chemosensitive patients $(32,33)$. Hu et al (27) demonstrated that lung metastasis was reduced following MTDH knockdown

The results of the MTT, Transwell and wound-healing assays in the present study revealed that cell growth and migration were inhibited in the MTDH-shRNA group compared with the blank and control-shRNA groups. MTDH is potentially a key regulator of tumor malignancy and is associated with the progression of certain types of cancer (34). A study by Hu et al (27) indicated that the therapeutic targeting of MTDH inhibited tumor growth and inhibited metastasis, which is 
A

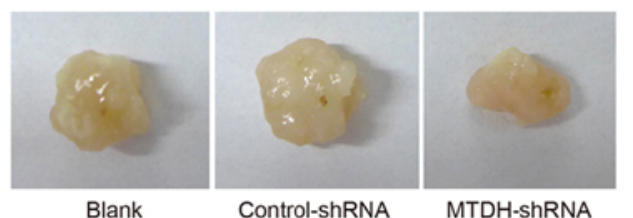

B

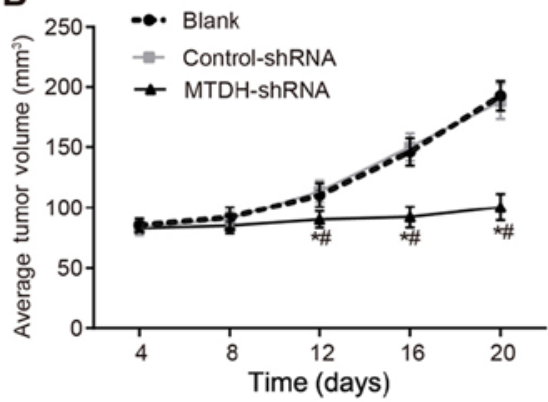

D

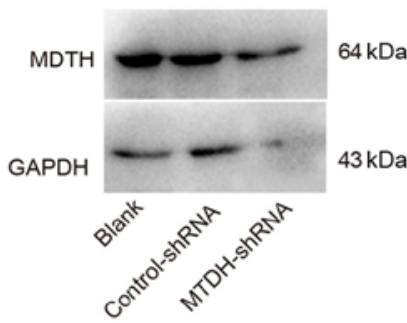

E

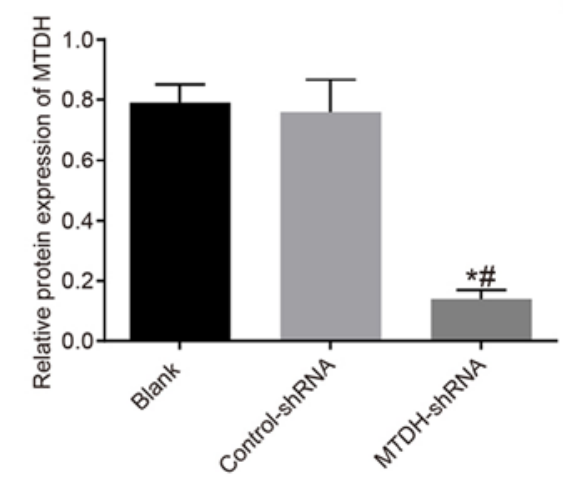

\section{C}

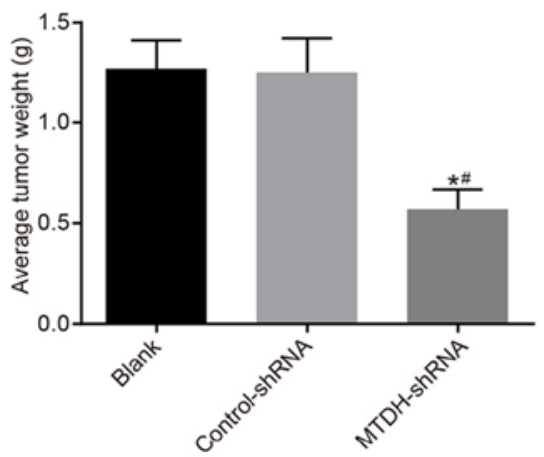

Figure 8. MTDH silencing increases radiosensitivity and inhibits ovarian cancer cell viability. (A) Images of representative tumors from each group; MTDH silencing lead to smaller (B) tumor volume and (C) tumor weight. (D) Western blot analysis was used to examine and (E) measure the relative expression of MTDH. *P $<0.05$ vs. MTDH-shRNA group; ${ }^{\#} \mathrm{P}<0.05$ vs. 0 Gy. Data are presented as the mean \pm standard deviation and were analyzed by one-way analysis of variance. The experiment was repeated three times. MTDH, metadherin; shRNA, short hairpin RNA.

consistent with the results of the present study. The expression of MTDH during developmental and differentiation processes is mediated by the phosphoinositide 3-kinase/Akt, nuclear factor- $\kappa \mathrm{B}$ and $\mathrm{Wnt} / \beta$-catenin signaling pathways $(26,27)$. The proliferation and invasion of cancer cells can be enhanced by activating these pathways (27). Furthermore, the development of hepatocellular carcinoma can be delayed by the microRNA-375-induced downregulation of MTDH (30). It has been reported that MTDH is involved in a number of physiological and pathological tumors, including brain tumors and neuroblastomas, whereas MTDH interference suppresses the proliferation and migration of these cells $(35,36)$. RNA interference contributes to sequence-specific gene silencing through double-stranded RNAs, which inhibit gene expression by degrading a specific mRNA (37). MTDH silencing in the present study was able to inhibit the proliferation and metastasis of SKOV3 ovarian cancer cells.

The colony-formation assay and flow cytometry results revealed that the radiosensitivity and apoptotic rate of SKOV3 cells were enhanced following MTDH silencing. Radioresistance is a common issue that results in treatment failure, and it has been reported that radioresistance is mediated by tumor-related genes affecting cellular processes $(38,39)$. A number of factors are associated with cancer risk and radiosensitivity, including alterations in DNA repair, cell cycle or apoptotic pathways (40). Previous evidence has revealed that the downregulation of MTDH can reduce the viability, colony formation and invasion of U87 human glioma cells and 9L rat gliosarcoma cells $(41,42)$. In addition, MTDH knockdown was shown to reduce radioresistance in colon cancer cell lines following irradiation (43). Cell viability and apoptosis were measured following treatment with specific interfering RNA, and it was reported that the downregulation of MTDH had no significant effects on cell cycle distribution, rather reducing cell viability via apoptosis (44). MTDH acts by interfering with protein translation via mRNA binding in the cytoplasm or by loading other mRNAs to the polysome (29). A study by Chang et al (45) revealed that cell apoptosis was significantly elevated in MTDH-knockdown groups compared with negative control groups. These results were supported by the in vivo experiment with nude mouse xenograft models in the present study, in which MTDH silencing enhanced the therapeutic efficacy of radiation and effectively inhibited tumor growth.

In conclusion, the results of the present study suggested that MTDH is expressed at a high level in patients with ovarian cancer. MTDH silencing inhibited the proliferation and metastasis of SKOV3 ovarian cancer cells and simultaneously induced apoptosis. Furthermore, MTDH silencing led to increased radiosensitivity of SKOV3 cells. Therefore, MTDH gene silencing may serve as a novel therapeutic strategy for the management of ovarian cancer. However, the effects of X-ray radiation on the expression of p53 and MDTH have not been investigated, and are to be the focus of future investigations. Gene expression is affected by various factors, and further investigations are required to confirm the aforementioned conclusions.

\section{Acknowledgements}

The authors are grateful to the reviewers for their critical comments on this manuscript. 


\section{Funding}

No funding was received.

\section{Availability of data and materials}

The datasets used and/or analyzed during the current study are available from the corresponding author on reasonable request.

\section{Authors' contributions}

JC conceived and designed the study. YJ and YMJ collated the data, designed and developed the database, performed data analyses and produced the initial draft of the manuscript. $\mathrm{ZHJ}$ obtained the results and validated them, YZ reviewed the results and discussions, $\mathrm{ZHI}$ and $\mathrm{YZ}$ revised the figures and tables and contributed to the revision of the manuscript. All authors have read and approved the final submitted manuscript.

\section{Ethics approval and consent to participate}

The present study was approved by the Ethics Committee of the Second Hospital of Jilin University (grant no. 201112006). Informed consent was obtained from patients or their guardians in accordance with the Declaration of Helsinki. The animal experiment was approved by the Animal Ethics Committee of the Second Hospital of Jilin University (no. 201201003), and the experiment strictly followed the National Institutes of Health guide for the care and use of laboratory animals.

\section{Patient consent for publication}

Not applicable.

\section{Competing interests}

The authors declare that they have no competing interests.

\section{References}

1. Meng Y, Hu J, Chen Y, Yu T and Hu L: Silencing MARCH1 suppresses proliferation, migration and invasion of ovarian cancer SKOV3 cells via downregulation of NF- $\mathrm{BB}$ and $\mathrm{Wnt} / \beta$-catenin pathways. Oncol Rep 36: 2463-2470, 2016.

2. Tan Y, Feng Q, Sun X, Xue M, Jiang N and Deng X: Effects of methylseleninic acid on cisplatin-resistant ovarian cancer cells (SKOV3/DDP) and the mechanisms. Zhong Nan Da Xue Xue Bao Yi Xue Ban 41: 1305-1311, 2016 (In Chinese).

3. Kaur A, Sultan SH, Murugaiah V, Pathan AA, Alhamlan FS, Karteris E and Kishore U: Human Clq induces apoptosis in an ovarian cancer cell line via tumor necrosis factor pathway. Front Immunol 7: 599, 2016

4. Musto A, Grassetto G, Marzola MC, Rampin L, Chondrogiannis S, Maffione AM, Colletti PM, Perkins AC, Fagioli G and Rubello D: Management of epithelial ovarian cancer from diagnosis to restaging: An overview of the role of imaging techniques with particular regard to the contribution of 18 F-FDG PET/CT. Nucl Med Commun 35: 588-597, 2014.

5. Holschneider CH and Berek JS: Ovarian cancer: Epidemiology, biology, and prognostic factors. Semin Surg Oncol 19: 3-10, 2000.

6. He M, Sun HG, Hao JY, Li YL, Yu JK, Yan YY, Zhao L, Li N, Wang Y, Bai XF, et al: RNA interference-mediated FANCF silencing sensitizes OVCAR3 ovarian cancer cells to adriamycin through increased adriamycin-induced apoptosis dependent on JNK activation. Oncol Rep 29: 1721-1729, 2013.
7. Wang NN, Zhao LJ, Wu LN, He MF, Qu JW, Zhao YB, Zhao WZ, Li JS and Wang JH: Mechanistic analysis of taxol-induced multidrug resistance in an ovarian cancer cell line. Asian Pac J Cancer Prev 14: 4983-4988, 2013.

8. Concin N, Zeillinger C, Stimpfel M, Schiebel I, Tong D, Wolff U, Reiner A, Leodolter S and Zeillinger R: p53-dependent radioresistance in ovarian carcinoma cell lines. Cancer Lett 150: 191-199, 2000.

9. Ma Y, Lu Y and Lu B: MicroRNA and long non-coding RNA in ovarian carcinoma: Translational insights and potential clinical applications. Cancer Invest 34: 465-476, 2016.

10. Cocco E, Deng Y, Shapiro EM, Bortolomai I, Lopez S, Lin K, Bellone S, Cui J, Menderes G, Black JD, et al: Dual-targeting nanoparticles for in vivo delivery of suicide genes to chemotherapyresistant ovarian cancer cells. Mol Cancer Ther 16: 323-333, 2017.

11. Shi $X$ and Wang X: The role of MTDH/AEG-1 in the progression of cancer. Int J Clin Exp Med 8: 4795-4807, 2015.

12. Wan L, Lu X, Yuan S, Wei Y, Guo F, Shen M, Yuan M, Chakrabarti R, Hua Y, Smith HA, et al: MTDH-SND1 interaction is crucial for expansion and activity of tumor-initiating cells in diverse oncogene- and carcinogen-induced mammary tumors. Cancer Cell 26: 92-105, 2014.

13. Wan L and Kang Y: Pleiotropic roles of AEG-1/MTDH/LYRIC in breast cancer. Adv Cancer Res 120: 113-134, 2013.

14. Guo F, Wan L, Zheng A, Stanevich V, Wei Y, Satyshur KA, Shen M, Lee W, Kang Y and Xing Y: Structural insights into the tumor-promoting function of the MTDH-SND1 complex. Cell Reports 8: 1704-1713, 2014.

15. Tan L, Qin H, Piao Y, Liu Z, Han Y, Song F and Xie X: Expression and clinical significance of MTDH and VEGF in triple-negative breast cancer. Zhonghua Zhong Liu Za Zhi 37: 827-832, 2015 (In Chinese).

16. Jia $\mathrm{X}$, Shan $\mathrm{C}, \mathrm{Xu} \mathrm{O}$ and Wang J: Expression and clinical significance of MTDH, HIF-1 $\alpha$ and TKTL1 in laryngeal carcinoma. Lin Chung Er Bi Yan Hou Tou Jing Wai Ke Za Zhi 29: 2133-2138, 2015 (In Chinese).

17. Zhao Y, Moran MS, Yang Q, Liu Q, Yuan C, Hong S and Kong B: Metadherin regulates radioresistance in cervical cancer cells. Oncol Rep 27: 1520-1526, 2012.

18. Gu X, Wang C, Wang X, Ma G, Li Y, Cui L, Chen Y, Zhao B and Li K: Efficient inhibition of human glioma development by RNA interference-mediated silencing of PAK5. Int J Biol Sci 11: 230-237, 2015.

19. Yiwei T, Hua H, Hui G, Mao M and Xiang L: HOTAIR interacting with MAPK1 regulates ovarian cancer skov3 cell proliferation, migration, and invasion. Med Sci Monit 21: 1856-1863, 2015.

20. Kanter M, Turan G, Usta C, Usta A, Esen HH, Tavlı L, Celik C, Demirkol Y and Kanter B: Survivin and cycline D1 expressions are associated with malignant potential in mucinous ovarian neoplasms. J Mol Histol 47: 145-152, 2016.

21. Steinbichler TB, Alshaimaa A, Maria MV, Daniel D, Herbert R, Jozsef D and Ira-Ida S: Epithelial-mesenchymal crosstalk induces radioresistance in HNSCC cells. Oncotarget 9: 3641-3652, 2017.

22. No authors listed: Summaries for patients. Screening for ovarian cancer: U.S. Preventive Services Task Force reaffirmation recommendation statement. Ann Intern Med 157: I-56, 2012.

23. Lee SG, Kang DC, DeSalle R, Sarkar D and Fisher PB: AEG-1/MTDH/LYRIC, the beginning: Initial cloning, structure, expression profile, and regulation of expression. Adv Cancer Res 120: $1-38,2013$.

24. Yuan C, Li X, Yan S, Yang Q, Liu X and Kong B: The MTDH $(-470 \mathrm{G}>\mathrm{A})$ polymorphism is associated with ovarian cancer susceptibility. PLoS One 7: e51561, 2012.

25. Emdad L, Hu B, Das SK, Sarkar D and Fisher PB: AEG-1-AKT2: A novel complex controlling the aggressiveness of glioblastoma. Mol Cell Oncol 2: e995008, 2015.

26. Emdad L, Lee SG, Su ZZ, Jeon HY, Boukerche H, Sarkar D and Fisher PB: Astrocyte elevated gene-1 (AEG-1) functions as an oncogene and regulates angiogenesis. Proc Natl Acad Sci USA 106: 21300-21305, 2009.

27. Hu G, Wei Y and Kang Y: The multifaceted role of MTDH/AEG-1 in cancer progression. Clin Cancer Res 15: 5615-5620, 2009.

28. Emdad L, Sarkar D, Su ZZ, Lee SG, Kang DC, Bruce JN, Volsky DJ and Fisher PB: Astrocyte elevated gene-1: Recent insights into a novel gene involved in tumor progression, metastasis and neurodegeneration. Pharmacol Ther 114: 155-170, 2007.

29. Haug S, Schnerch D, Halbach S, Mastroianni J, Dumit VI, Follo M, Hasenburg A, Köhler M, Dierbach H, Herzog S, et al: Metadherin exon 11 skipping variant enhances metastatic spread of ovarian cancer. Int J Cancer 136: 2328-2340, 2015. 
30. He XX, Chang Y, Meng FY, Wang MY, Xie QH, Tang F, Li PY, Song YH and Lin JS: MicroRNA-375 targets AEG-1 in hepatocellular carcinoma and suppresses liver cancer cell growth in vitro and in vivo. Oncogene 31: 3357-3369, 2012.

31. Zhou B, Yang J, Shu B, Liu K, Xue L, Su N, Liu J and Xi T: Overexpression of astrocyte-elevated gene-1 is associated with ovarian cancer development and progression. Mol Med Rep 11: 2981-2990, 2015

32. Li C, Chen K, Cai J, Shi QT, Li Y, Li L, Song H, Qiu H, Qin Y and Geng JS: Astrocyte elevated gene-1: A novel independent prognostic biomarker for metastatic ovarian tumors. Tumour Biol 35: 3079-3085, 2014

33. Sarkar D and Fisher PB: AEG-1/MTDH/LYRIC: Clinical significance. Adv Cancer Res 120: 39-74, 2013.

34. Huang Y and Li LP: Progress of cancer research on astrocyte elevated gene-1/Metadherin (Review). Oncol Lett 8: 493-501, 2014

35. Wang Y, Zhang W, Zhu X, Wang Y, Mao X, Xu X and Wang Y: Upregulation of AEG-1 Involves in Schwann Cell Proliferation and Migration After Sciatic Nerve Crush. J Mol Neurosci 60 248-257, 2016

36. Yao Y, Gu X, Liu H, Wu G, Yuan D, Yang X and Song Y: Metadherin regulates proliferation and metastasis via actin cytoskeletal remodelling in non-small cell lung cancer. Br J Cancer 111: 355-364, 2014

37. Park JB, Chang DG, Oh SY and Park EY: Effect of RNA Interference-Mediated Suppression of p75 on the Viability of Rat Notochordal Cells. Asian Spine J 10: 985-992, 2016.

38. Zhu R, Li W, Xu Y, Wan J and Zhang Z: Upregulation of BTG1 enhances the radiation sensitivity of human breast cancer in vitro and in vivo. Oncol Rep 34: 3017-3024, 2015.
39. Huerta S, Gao X, Dineen S, Kapur P, Saha D and Meyer J: Role of p53, Bax, p21, and DNA-PKcs in radiation sensitivity of HCT-116 cells and xenografts. Surgery 154: 143-151, 2013.

40. Hornhardt S, Rößler U, Sauter W, Rosenberger A, Illig T, Bickeböller H, Wichmann HE and Gomolka M: Genetic factors in individual radiation sensitivity. DNA Repair (Amst) 16: 54-65, 2014.

41. Emdad L, Sarkar D, Lee SG, Su ZZ, Yoo BK, Dash R, Yacoub A, Fuller CE, Shah K, Dent P, et al: Astrocyte elevated gene-1: A novel target for human glioma therapy. Mol Cancer Ther 9: 79-88, 2010.

42. Awasthi S, Singhal SS, Awasthi YC, Martin B, Woo JH, Cunningham CC and Frankel AE: RLIP76 and cancer. Clin Cancer Res 14: 4372-4377, 2008.

43. Gnosa S, Zhang H, Brodin VP, Carstensen J, Adell G and Sun XF: AEG-1 expression is an independent prognostic factor in rectal cancer patients with preoperative radiotherapy: A study in a Swedish clinical trial. Br J Cancer 111: 166-173, 2014.

44. Nikpour M, Emadi-Baygi M, Fischer U, Niegisch G, Schulz WA and Nikpour P: MTDH/AEG-1 contributes to central features of the neoplastic phenotype in bladder cancer. Urol Oncol 32: 670-677, 2014

45. Chang Y, Li B, Xu X, Shen L, Bai H, Gao F, Zhang Z and Jonas JB: Lentivirus-mediated knockdown of astrocyte elevated gene-1 inhibits growth and induces apoptosis through MAPK pathways in human retinoblastoma cells. PLoS One 11: e0148763, 2016. 